\title{
Zinc Oxide Coated Carbon Dot Nanoparticles as Electron Transport Layer for Inverted Polymer Solar Cells
}

Wensheng Zhao, a,b Lingpeng Yan, ${ }^{\mathrm{a}, \mathrm{b}, \mathrm{c} *}$ Huimin Gu, ${ }^{\mathrm{a}, \mathrm{b}}$ Zerui Li, ${ }^{\mathrm{b}}$ Yaling Wang, ${ }^{\mathrm{e}}$

Qun Luo, ${ }^{\mathrm{b}}$ Yongzhen Yang, ${ }^{\text {a* }}$ Xuguang Liu, ${ }^{\mathrm{c}}$ Hua Wang, ${ }^{\mathrm{a}, \mathrm{d}}$ Chang-Qi Ma ${ }^{\mathrm{b} *}$

${ }^{a}$ Key Laboratory of Interface Science and Engineering in Advanced Materials, Ministry of Education,

Taiyuan University of Technology, Taiyuan 030024, P. R. China

${ }^{b}$ Printed Electronics Research Center, Suzhou Institute of Nano-Tech and Nano-Bionics, Chinese Academy of Sciences (CAS), Suzhou 215123, P. R. China

${ }^{\mathrm{c}}$ Institute of New Carbon Materials, Taiyuan University of Technology, Taiyuan 030024, P. R. China.

${ }^{d}$ College of Textile Engineering, Taiyuan University of Technology, Taiyuan 030600, PR China

${ }^{\mathrm{e}}$ School of Energy and Power Engineering, North University of China, Taiyuan, 030051 P. R. China 


\section{Support Information}

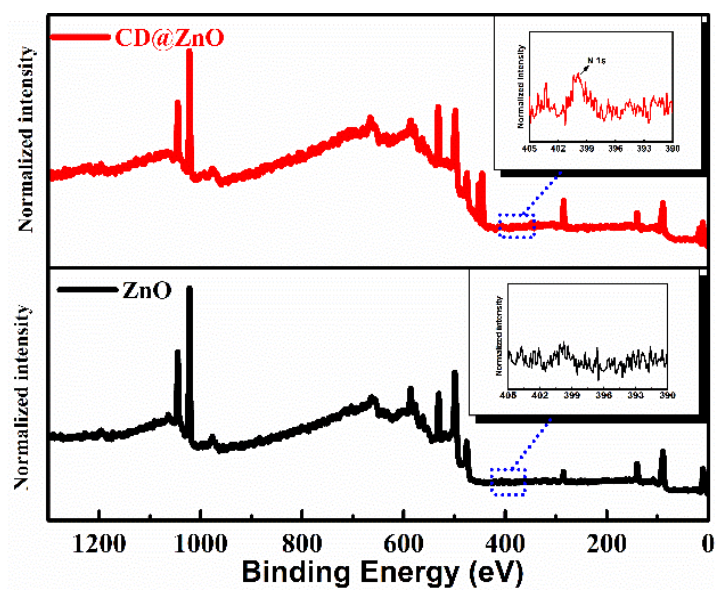

Figure S1. XPS spectra of $\mathrm{ZnO}$ and $\mathrm{CD} @ \mathrm{ZnO}$, the insets are enlarged view of $\mathrm{N} 1 \mathrm{~s}$.

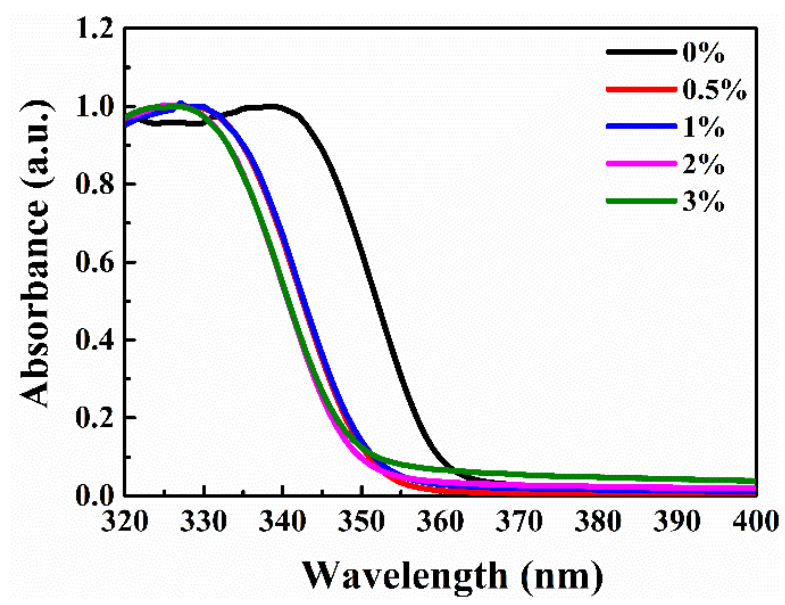

Figure S2. UV-vis absorption spectra of ZnO and CD@ZnO.

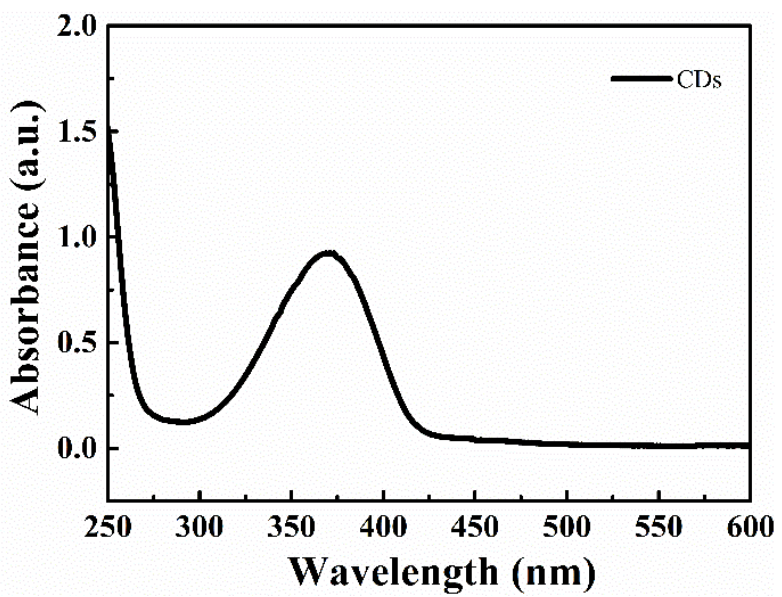

Figure S3. UV-vis absorption spectrum of CDs. 


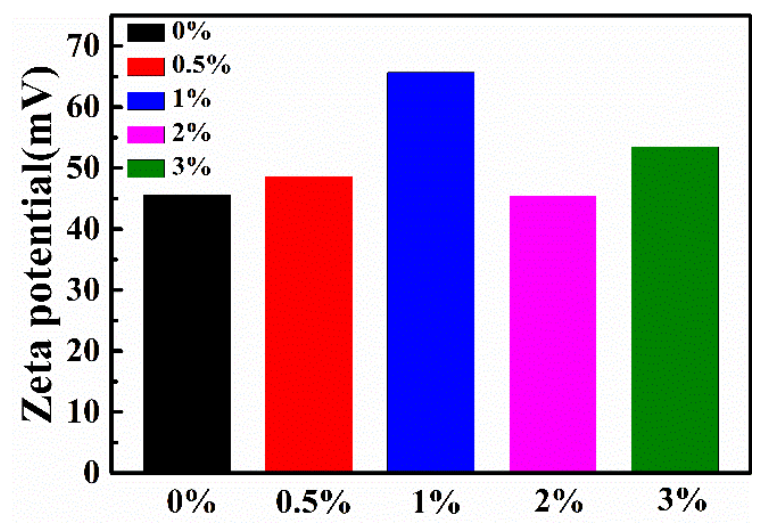

Figure S4. Zeta potentials of $\mathrm{ZnO}$ and $\mathrm{CD} @ \mathrm{ZnO}$ (excited at $3.4 \mathrm{~V}$ ).
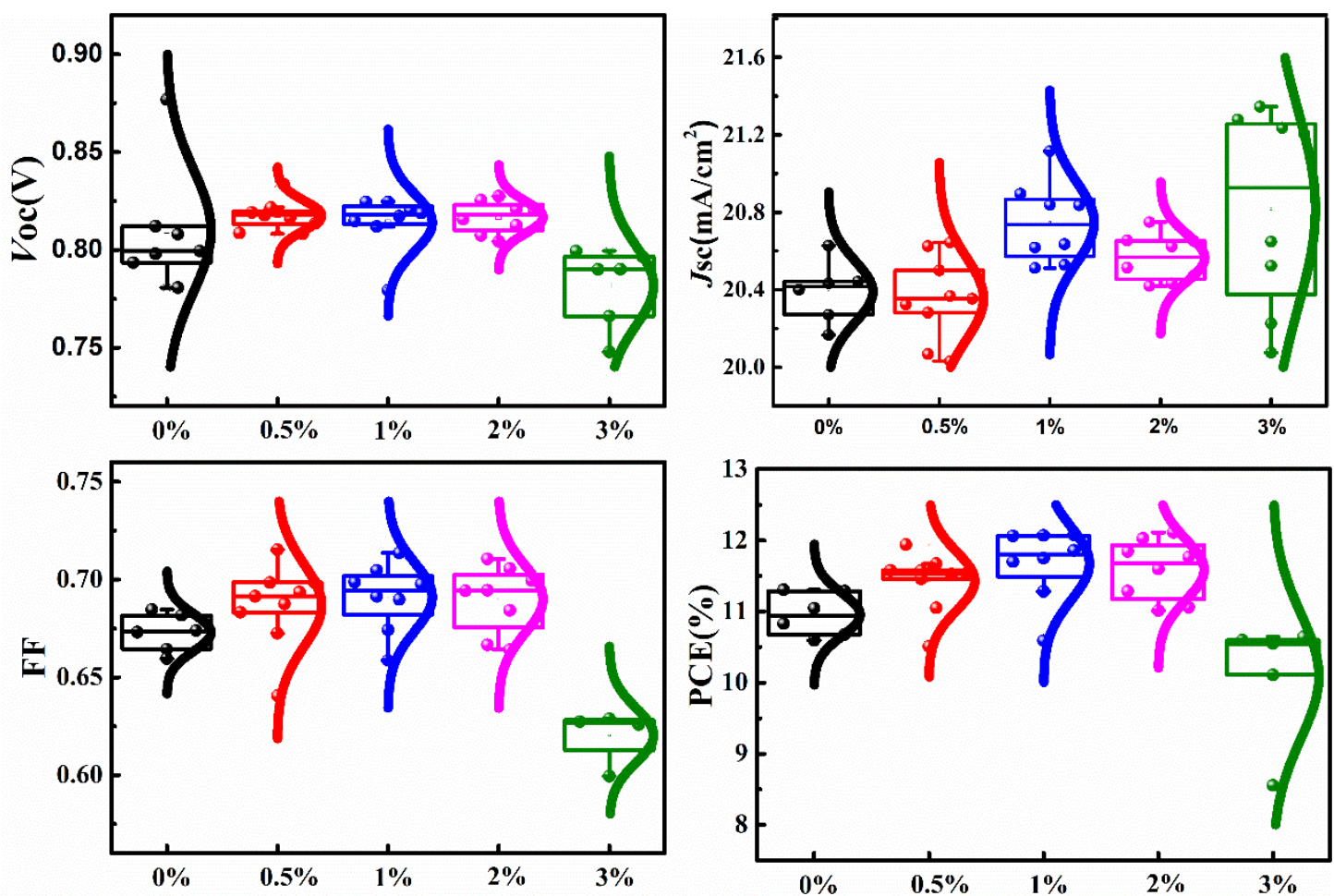

Figure S5. Performance statistical data for the devices with ZnO and CD@ZnO ETLs represented as a standard box plot shown in (a) $V_{\mathrm{OC}}$, (b) $J_{\mathrm{SC}}$, (c) FF, (d) PCE of different of CD@ZnO-based PSCs under AM 1.5G $100 \mathrm{~mW} / \mathrm{cm}^{2}$ simulated solar light. 

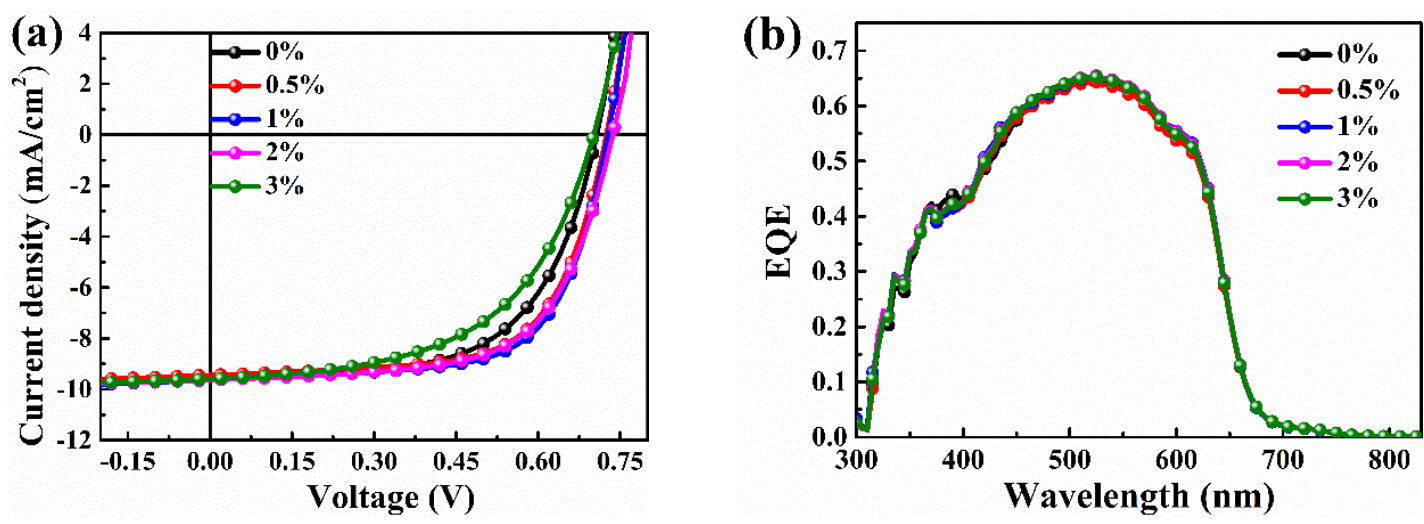

Figure S6. (a) $J-V$ curves of P3HT:bis-PCBM inverted polymer solar cells with pristine $\mathrm{ZnO}$ and

CD@ZnO ETLs. (b) EQE spectra of pristine ZnO and CD@ZnO ETLs solar cells.

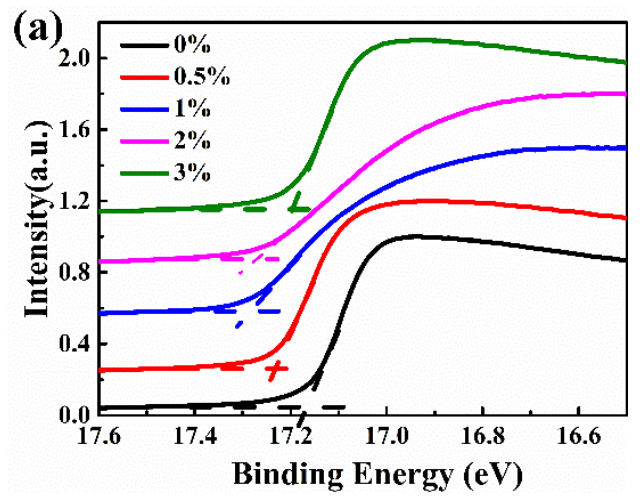

(b)

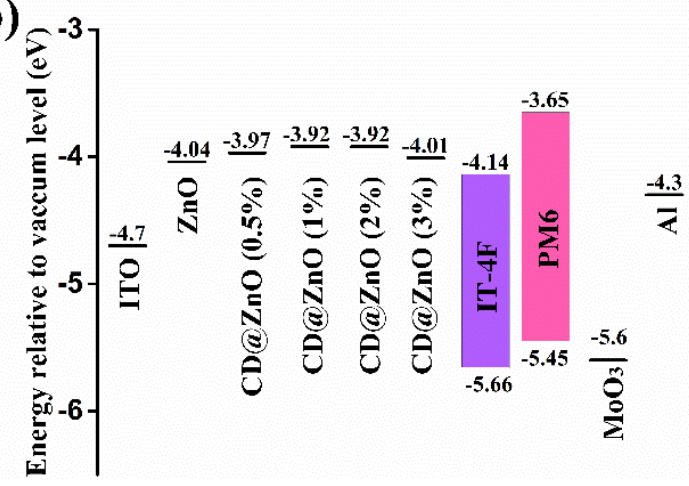

Figure S7 (a) UPS spectra of the pristine ZnO and CD@ZnO. (b) Energy level diagram of the PSCs. 

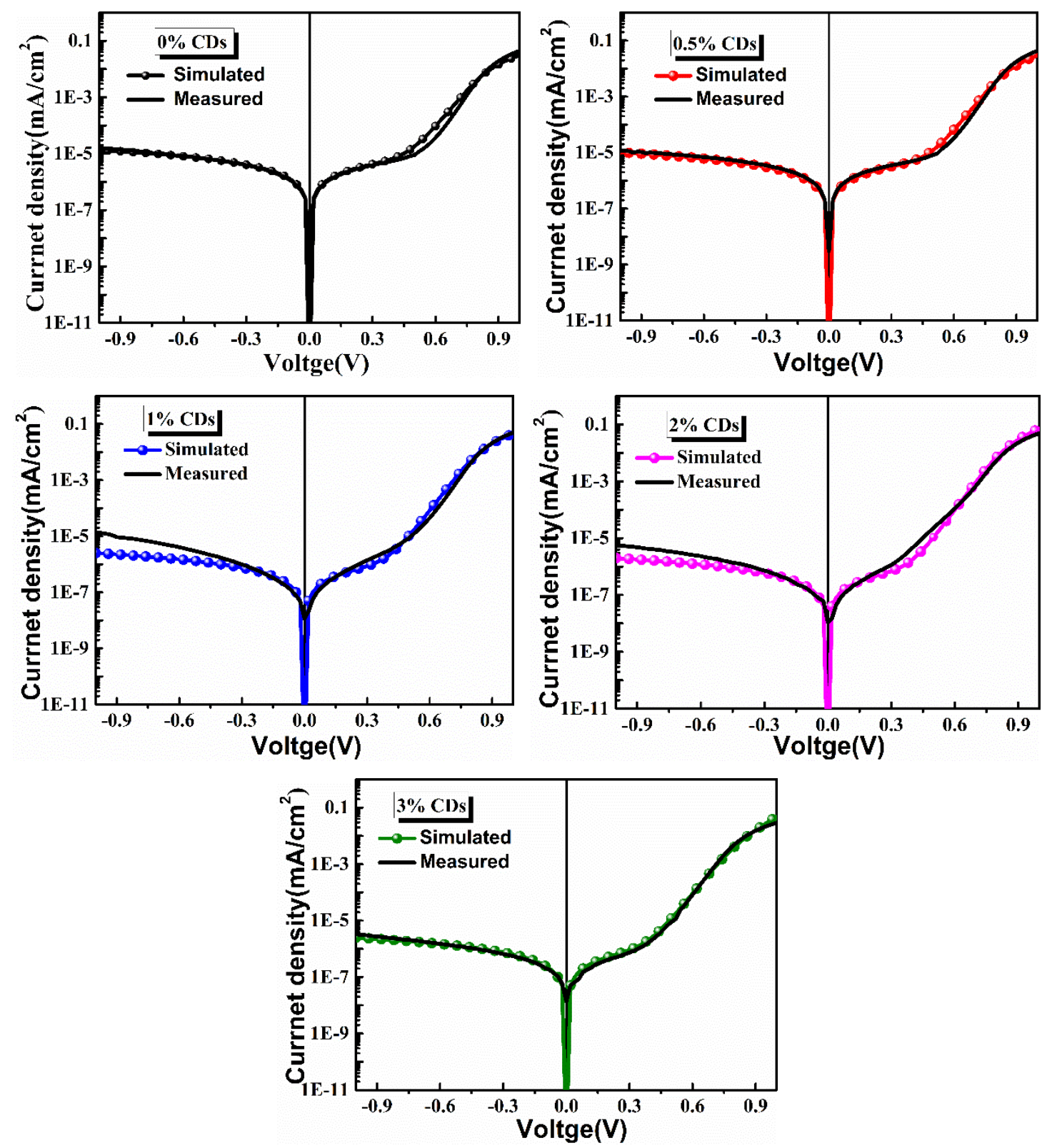

Figure S8. The measured and the simulated dark $J-V$ curves of PM6:IT-4F devices. 

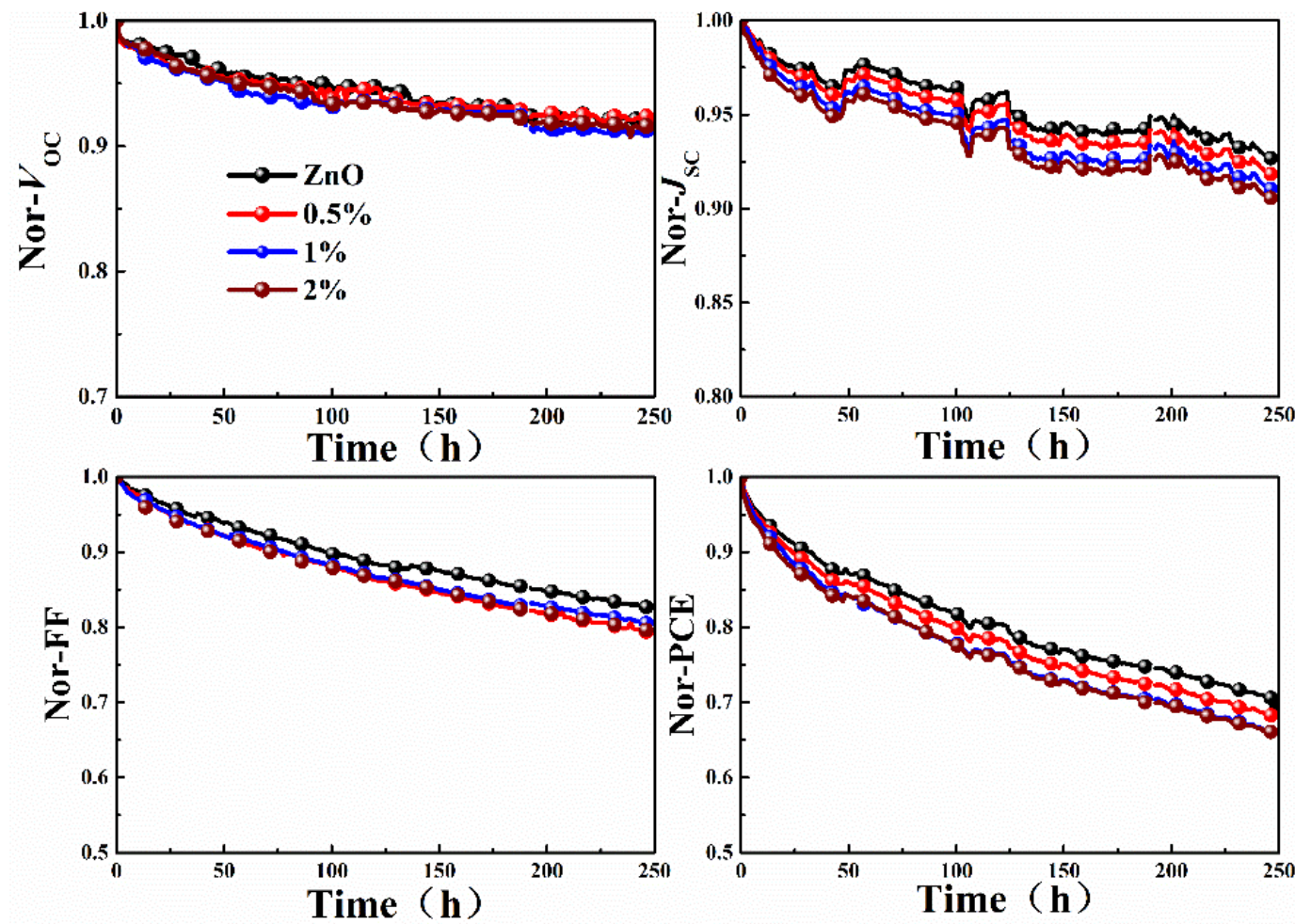

Figure S9. Normalized (a) $V_{\mathrm{OC}}$, (b) $J_{\mathrm{SC}}$, (c) FF, and (d) PCE decay of the PM6:IT-4F inverted solar cells using ZnO and CD@ZnO ETLs.

Table S1 Device PCE of the inverted polymer solar cells using pristine ZnO and CD@ZnO as

ETLs and using P3HT:bis-PCBM as the active layers

\begin{tabular}{ccccc}
\hline $\mathrm{m}(\mathrm{CDs}): \mathrm{m}(\mathrm{ZnO})$ & $V_{\mathrm{OC}}(\mathrm{V})$ & $J_{\mathrm{SC}}\left(\mathrm{mA} / \mathrm{cm}^{2}\right)$ & $\mathrm{FF}$ & $\mathrm{PCE}(\%)(\mathrm{av} \mathrm{PCE} \pm \mathrm{SD})$ \\
\hline $0 \%$ & 0.70 & 9.51 & 0.62 & $4.13(4.03 \pm 0.03)$ \\
$0.5 \%$ & 0.72 & 9.44 & 0.65 & $4.42(4.30 \pm 0.02)$ \\
$1 \%$ & 0.73 & 9.63 & 0.66 & $4.64(4.54 \pm 0.07)$ \\
$2 \%$ & 0.74 & 9.64 & 0.63 & $4.49(4.43 \pm 0.03)$ \\
$3 \%$ & 0.70 & 9.60 & 0.55 & $3.70(3.68 \pm 0.04)$ \\
\hline
\end{tabular}

The standard deviation was calculated over 8 individual devices. 